\title{
ÉTUDE DE L'ÉVAPOTRANSPIRATION RÉELLE DE QUATRE PEUPLEMENTS FORESTIERS DANS L'EST DE LA FRANCE
}

\author{
G. AUSSENAC \\ avec la collaboration technique de J.-M. Desjeunes et F. Willm. \\ Station de Sylviculture et de Production, \\ Centre national de Recherches forestières, I.N.R.A., \\ 54 - Nancy
}

\section{RÉSUMÉ}

L'évapotranspiration réelle (E.T.R.) de quatre peuplements forestiers : Pins sylvestres (Pinus sylvestris), Épicéa (Picea abies), Sapin de Vancouver (Abies grandis), Feuillus (Fagus silvatica + Carpinus betulus) a été étudiée pendant 3 ans, de 1967 à 1969.

Ces peuplements à structure simple (futaie) sont installés en terrain plat sur des sols bruns lessivés marmorisés, présentant un horizon d'engorgement (à $60 \mathrm{~cm}$ de profondeur). L'étude a consisté à évaluer l'évapotranspiration des peuplements pendant des périodes pour lesquelles au départ (début de la saison de végétation) et à la fin (fin de la saison de végétation) le sol est à la capacité au champ. L'équation de base est la suivante : $\mathrm{E} \mathrm{T} \mathrm{R}=\mathrm{P}_{i}-\mathrm{D} \pm \Delta \mathrm{N}$.

$$
\begin{aligned}
& \mathrm{P}_{i}=\text { précipitations incidentes tombant sur le peuplement. } \\
& \mathrm{D}=\text { drainage latéral et profond. } \\
& \mathrm{N}=\text { variation du stock d'eau des nappes perchées. }
\end{aligned}
$$

Le drainage a été évalué en étudiant les fluctuations diurnes et nocturnes des nappes. La fluctuation des nappes a été suivie à l'aide de tubes piezométriques. Les résultats obtenus montrent qu'il y a des différences entre les peuplements. En particulier les Épicéas évapotranspirent moins que les autres. Ceci peut être expliqué par des différences d'âge, de structure et de situation par rapport à l'environnement (advection). Il est certain que les phénomènes d'interception des précipitations jouent également un rôle important, car la perte nette peut être très variable suivant les essences et la structure des peuplements.

L'étude effectuée montre que l'évapotranspiration réelle dépasse ou est égale à l'évapotranspiration potentielle évaluée par la formule de Bouchet. Finalement, les différences relativement faibles paraissent indiquer que sous nos climats tempérés humides et sur sols identiques aux nôtres, les peuplements forestiers comparables (âge, structure, etc.) à couvert fermé ont des évapotranspirations réelles voisines.

\section{I. - INTRODUCTION}

L'évaluation de l'évapotranspiration réelle des peuplements forestiers est du plus grand intérêt pour la sylviculture, que l'on considère le problème des la croissance des arbres et des peuplements ou celui de l'influence de la forêt sur l'économie de l'eau dans la nature.

Dans un pays comme la France, la définition des relations forêt-cycle de l'eau est rendue urgente par la restructuration de l'agriculture avec pour conséquence des reboisements et des déboisements considérables, la transformation rapide de la forêt (enrésinement des 
TABLEAU 1 - TABLE 1

Caractéristiques des 4 peuplements étudiés

Conditions of the forest sites

\begin{tabular}{|c|c|c|c|c|c|c|c|}
\hline Peuplement expérimental & Situation & $\begin{array}{c}\text { Age } \\
\text { (années) }\end{array}$ & $\begin{array}{c}\text { Nombre } \\
\text { d'arbres/ha }\end{array}$ & $\begin{array}{c}\text { Hauteur } \\
\text { moyenne }(\mathrm{m})\end{array}$ & $\begin{array}{l}\text { Diamètre moyen } \\
(\mathrm{cm} \text { à } 130 \mathrm{~m})\end{array}$ & $\begin{array}{l}\text { Accroissement } \\
\text { moyen annuel } \\
\mathrm{m} 3 / \text { ha }(1)\end{array}$ & $\begin{array}{l}\text { Accroissement } \\
\text { courant } \\
\mathrm{m} 3 / \mathrm{ha} / \mathrm{an}\end{array}$ \\
\hline $\begin{array}{l}\text { Épicéa commun } \\
\text { Picea abis L. (Karsten,) . ..... }\end{array}$ & $\begin{array}{l}\text { ARBoretum } \\
\text { d'Amance }\end{array}$ & 24 & 2160 & 12,5 & 12,0 & 9,6 & 25,4 \\
\hline $\begin{array}{l}\text { Pin Sylvestre } \\
\text { Pinus silvestris L. . . . . . . . }\end{array}$ & $\begin{array}{l}\text { ARBORETUM } \\
\text { d'Amance }\end{array}$ & 29 & 1520 & 13,0 & 17,0 & 5,5 & 11,2 \\
\hline $\begin{array}{l}\text { Sapin de Vancouver } \\
\text { Abis grandis Douglas Lindt } \ldots\end{array}$ & $\begin{array}{l}\text { ARBORETUM } \\
\text { d'Amance }\end{array}$ & 33 & 620 & 23,0 & 30,3 & 12,5 & 30,0 \\
\hline $\begin{array}{l}\text { Feuillus } \\
\text { Fagus silvatica } L . \\
\text { Carpinus betulus } L . \ldots \ldots \ldots \ldots\end{array}$ & $\begin{array}{l}\text { Forêt domaniale } \\
\text { d'Amance } \\
\text { P. } 48\end{array}$ & 30 & 1300 & 12,5 & 8,7 & & 7,8 \\
\hline
\end{tabular}

(1) production moyenne par ha et par an depuis l'origine. 
taillis sous futaie, substitution aux essences naturelles d'espèces exotiques : Sapin de Vancouver, Sapin de Douglas, etc.).

Un programme de recherches a été lancé sur ce thème dans quatre peuplements forestiers dans l'est de la France. Dans une première série d'articles (AussenAC 1968, AussenAC 1970), ont été présentés les résultats relatifs au devenir des précipitations incidentes.

Le présent travail expose maintenant l'étude de l'évapotranspiration réelle de ces peuplements. L'expérimentation a été réalisée à $15 \mathrm{~km}$ à l'est de Nancy, en forêt domaniale d'Amance, (altitude $250 \mathrm{~m}$, latitude $48^{\circ} 44^{\prime} \mathrm{N}$, longitude $06^{0} 14^{\prime} \mathrm{E}$ ).

\section{II. - DISPOSITIF EXPÉRIMENTAL ET MÉTHODES UTILISÉES}

\section{1. - Les peuplements étudiés.}

Les peuplements étudiés (tableau $n^{\circ} 1$ ) présentent une structure simple : futaie équienne à un seul étage, sans strate arbustive, mais une strate herbacée bien développée existe dans le peuplement de Pin sylvestre. Ils sont situés sur un terrain non accidenté sur un plateau et ils sont suffisamment proches pour qu'ils puissent être considérés comme étant placés dans des conditions climatiques générales identiques (figure $\mathrm{n}^{\circ} 1$ ). Ces peuplements sont installés sur des sols bruns lessivés marmorisés (horizon d'engorgement à une profondeur moyenne de $60 \mathrm{~cm}$ ) qui se sont développés sur des marnes

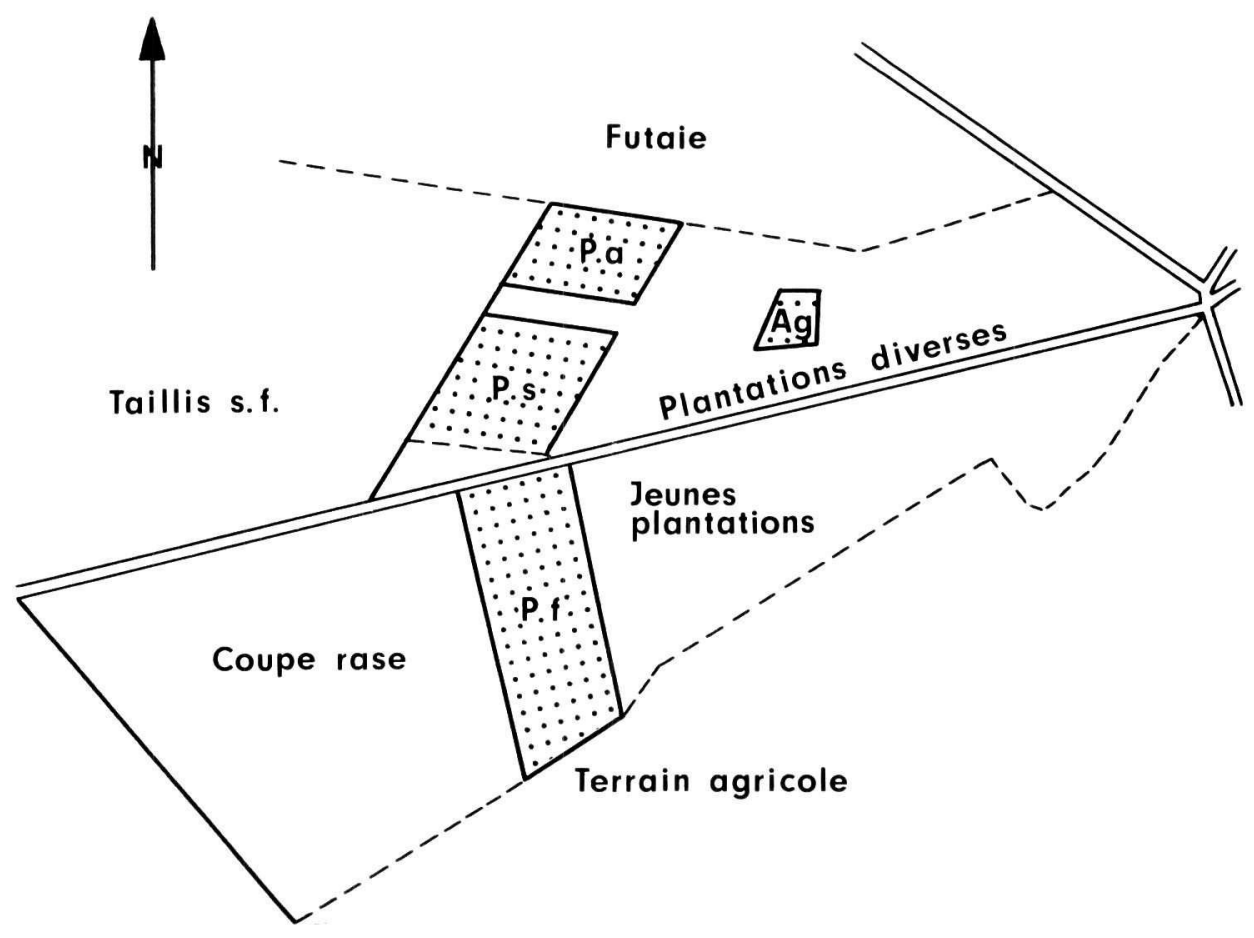

Echelle : $1 / 10000$

FIG. 1. - Plan de situation des quatres peuplements étudiés

FIG. 1. - Situat on of four stands 
liasiques recouvertes de limons. Pour ces sols, la réserve d'eau utile $(\mathrm{pF}=2,5 \mathrm{à} \mathrm{pF}=4,2)$ est voisine de $100 \mathrm{~mm}$.

\section{2. - Les méthodes utilisées.}

La caractéristique principale du substrat sur lesquels sont installés les peuplements étudiés est l'existence d'un horizon d'engorgement très compact développé sur des marnes liasiques. La présence de cet horizon imperméable a pour conséquence d'une part de limiter l'enracinement des arbres aux 70 premiers centimètres et d'autre part en freinant le drainage profond, de permettre le développement de nappes perchées temporaires. A partir de cette constatation, il est possible d'écrire que pour une période donnée :

$$
\begin{aligned}
\text { (1) } \mathrm{Pi}=\mathrm{ETR}+\mathrm{D} \pm \Delta \mathrm{N} \text { avec Pi : précipitations incidentes } \\
\mathrm{ETR}: \text { : évapotranspiration réelle } \\
\mathrm{D}: \text { drainage profond et latéral } \\
\Delta \mathrm{N}: \text { variation du stock d'eau des } \\
\text { nappes perchées. }
\end{aligned}
$$

La méthode utilisée pour évaluer l'évapotranspiration réelle des peuplements découle de la formule précédente en considérant des périodes pour lesquelles au départ (début de la saison de végétation) et à la fin (fin de la saison de végétation) le sol est à la capacité au champ. Il vient alors :

$$
\text { (2) } \mathrm{ETR}=\mathrm{Pi}-\mathrm{D} \pm \Delta \mathrm{N} \text {. }
$$

A partir de là, il est nécessaire d'évaluer les paramètres $\mathrm{Pi}, \mathrm{D}$ et $\Delta \mathrm{N}$. En ce qui concerne les précipitations, les mesures effectuées ont porté sur les précipitations incidentes $\mathrm{Pi}$, mais aussi sur les précipitations arrivant directement au sol ou ruisselant le long des troncs (AusSENAC 1968, AusseNAC 1970). Les précipitations incidentes ( $\mathrm{Pi})$ ont été évaluées à l'aide de pluviomètres installés sur une

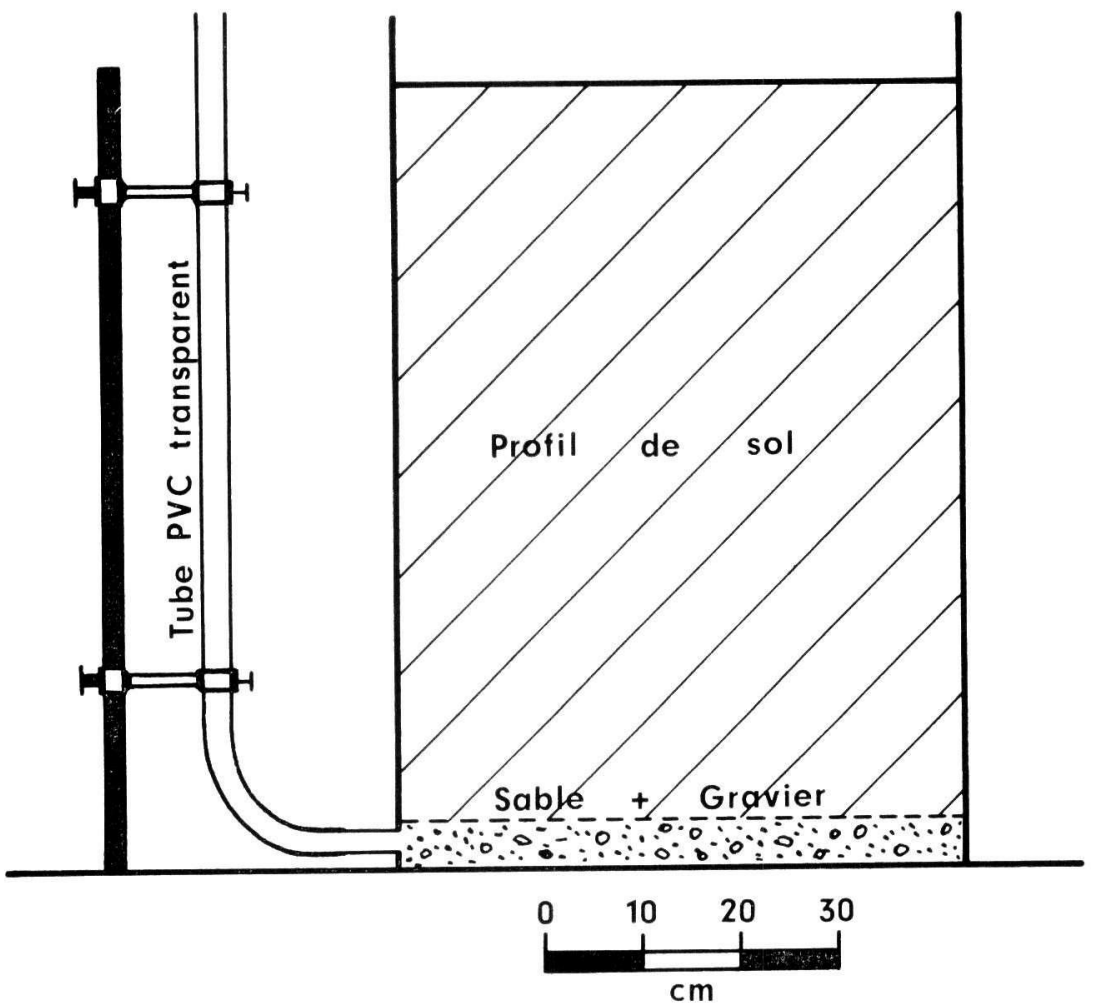

FIG. 2. - Croquis du dispositif utilisé pour évaluer pour une hauteur de nappe considérée son équivalent en eau

FIG. 2. - Sketch of apparatus utilised for evaluation of water equivalent of the water table 
tour au-dessus des peuplements. Des mesures effectuées à 1,50 $\mathrm{m}$ au-dessus du sol donnent des valeurs légèrement plus élevées (différences journalières $\leqslant 0,5 \mathrm{~mm}$ ). Ces écarts peuvent s'expliquer par des variations du coefficient de captation des pluviomètres avec la vitesse du vent. Il est donc possible que l'évaluation de Pi présente une erreur par défaut.

Pour ce qui est de la variation du stock d'eau des nappes perchées et du drainage, les fluctuations des nappes ont été étudiées à l'aide de tubes piézométriques installés dans le sol jusque dans l'horizon d'engorgement. Les piézomètres sont constitués de tubes de $80 \mathrm{~mm}$ de diamètre en polyvinyle. Une technique identique a été décrite par LeVY (1969).

\section{TABLEAU 2 - TABLE 2}

Variation de la porosité totale (en \% du volume) avec la profondeur Variations of total porosity on the four sites

\begin{tabular}{c|c|c|c|c}
\hline \hline $\begin{array}{c}\text { Profondeur } \\
(\mathrm{cm})\end{array}$ & Épicéa & Pin sylvestre & Sapin de Vancouver & Peuplement feuillu \\
& & & & \\
& & & & \\
10 & 58,2 & 56,3 & 56,0 & 59,0 \\
20 & 55,0 & 52,9 & 53,1 & 57,9 \\
30 & 51,9 & 51,8 & 51,0 & 53,1 \\
40 & 50,8 & 49,9 & 48,6 & 51,3 \\
50 & 52,5 & 46,7 & 51,2 & 48,3 \\
60 & 50,9 & 45,9 & 50,9 & 48,0 \\
70 & 50,4 & 48,0 & 50,3 & 47,9 \\
\hline
\end{tabular}

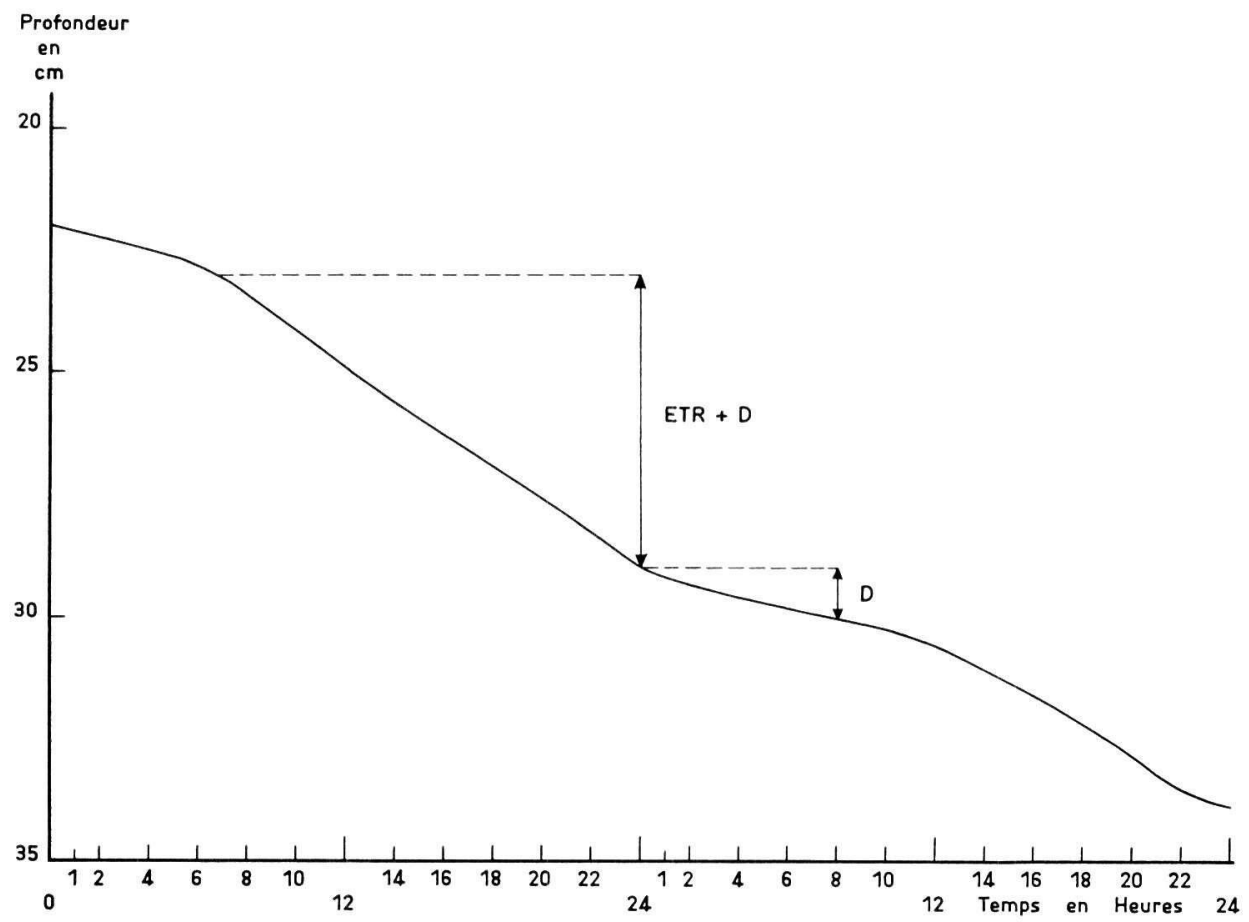

FIG. 3. - Exemple de fluctuation de la profondeur de la nappe perchée du 18 avril 1970 au 19 avril 1970 FIG. 3. - Example of fluctuation of the water table depth 

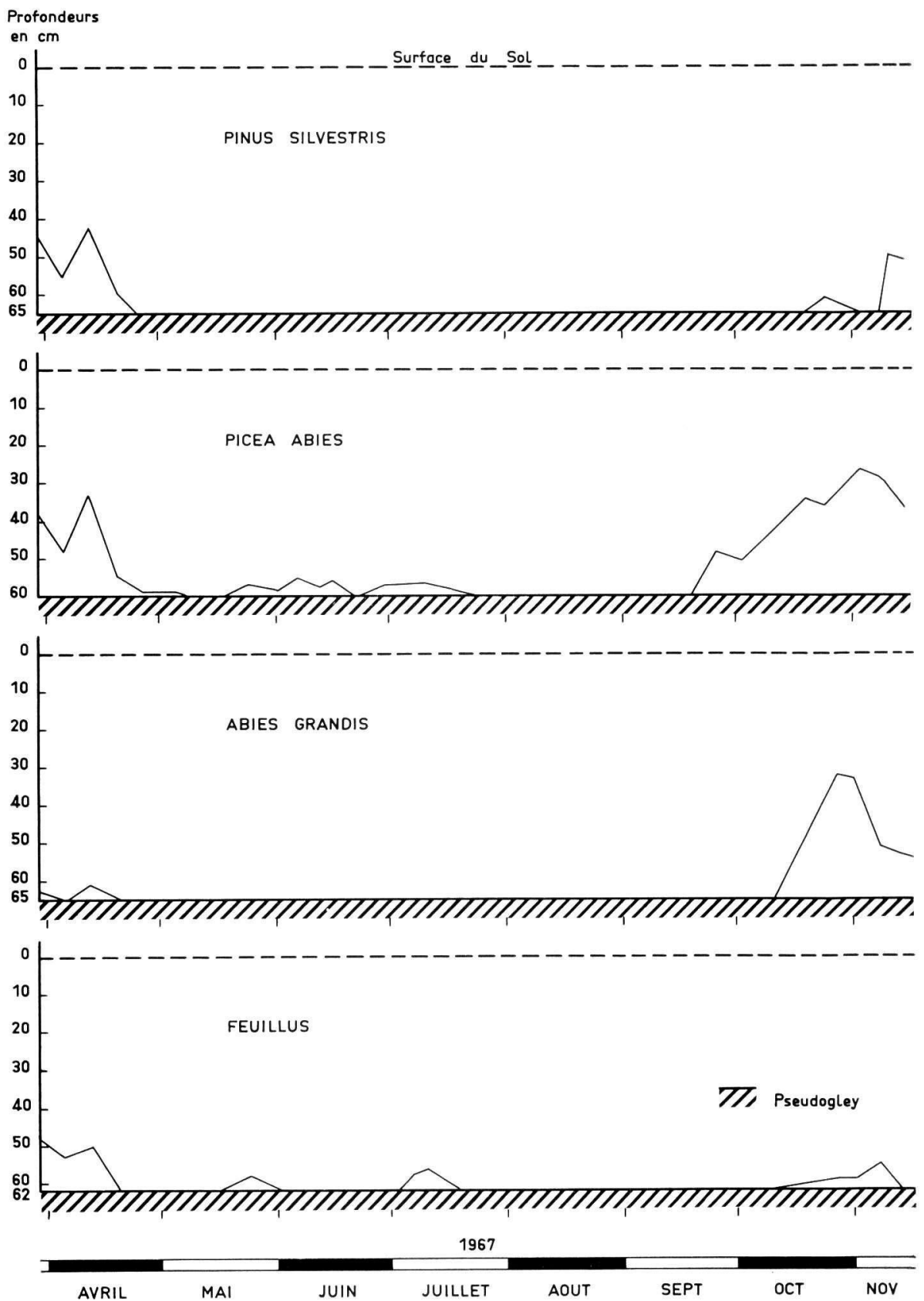

FIG. 4. - Fluctuation de la profondeur des nappes perchées pendant la saison de végétation en 1967 FIG. 4. - Fluctuation of the water table depth during the period of vegetation in 1967 

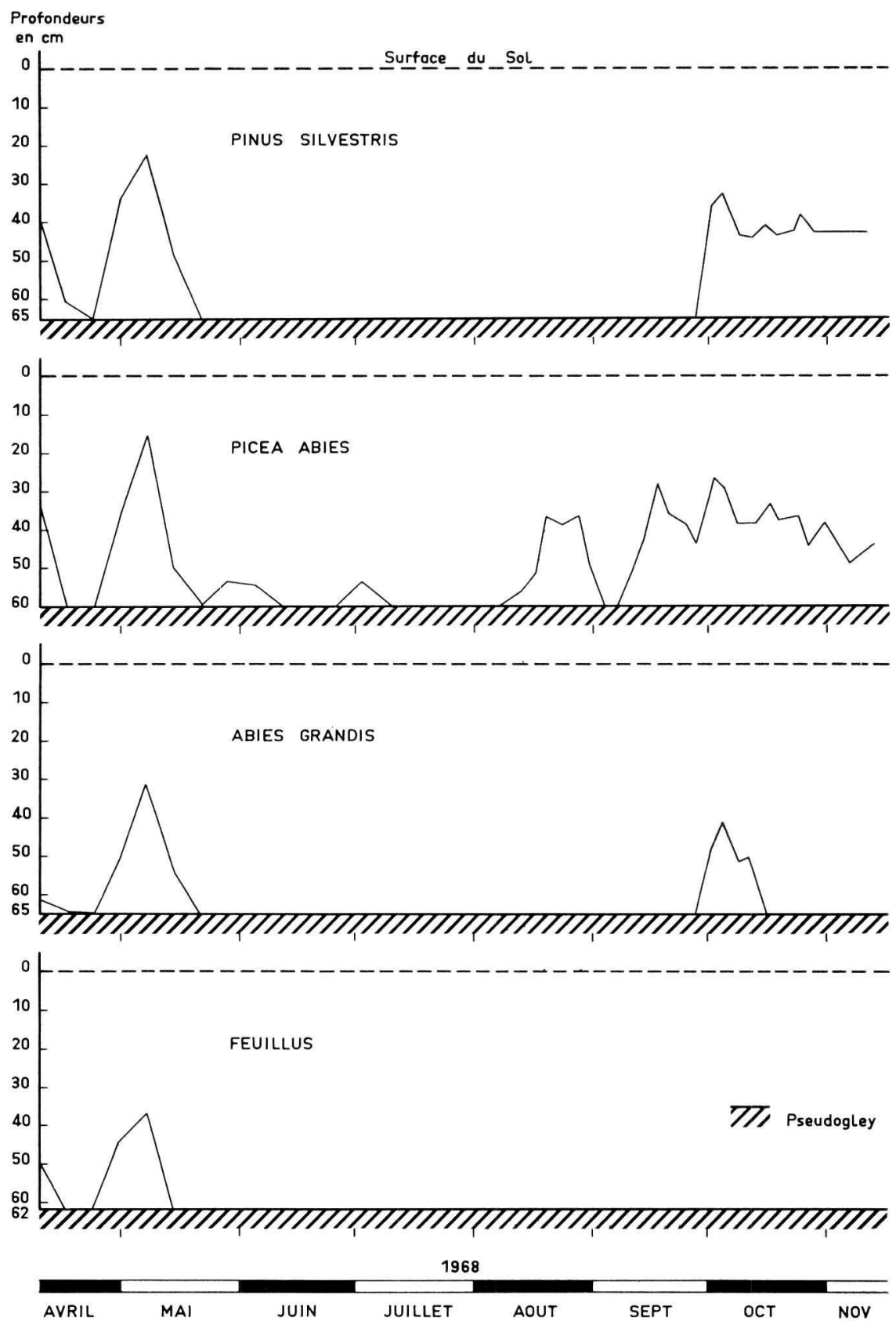

FIG. 5. - Fluctuation de la profondeur des nappes perchées pendant la saison de végétation en 1968 FIG. 5. - Fluctuation of the water table depth during the period of vegetation in 1968 


\section{Profondeurs}
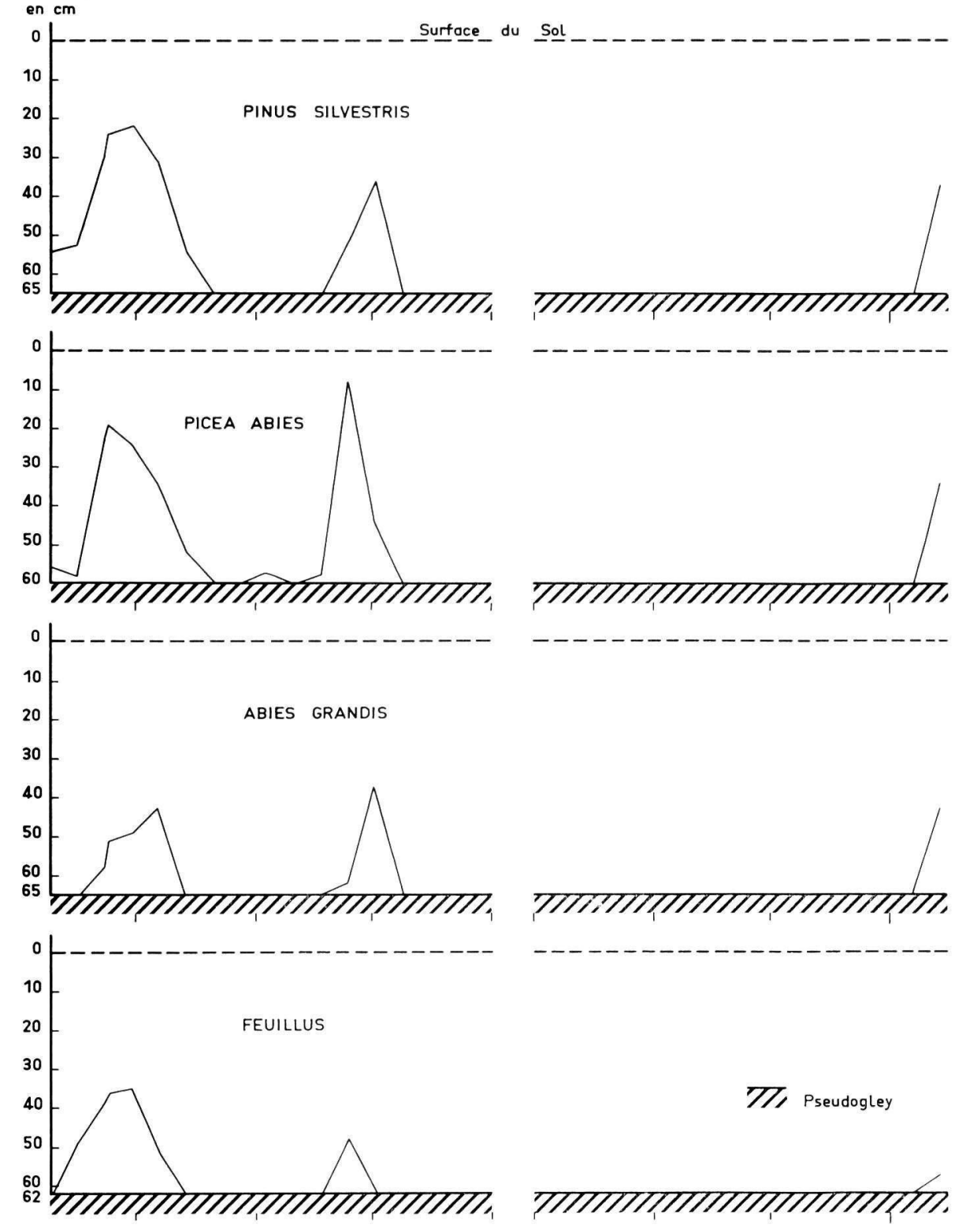

7) Pseudogley

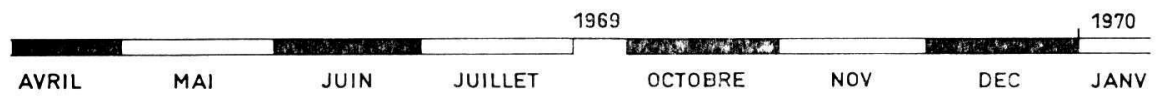

Fig. 6. - Fluctuation de la profondeur des nappes perchées pendant la saison de végétation en 1969 FIG. 6. - Fluctuation of the water table depth during the period of vegetation in 1969 

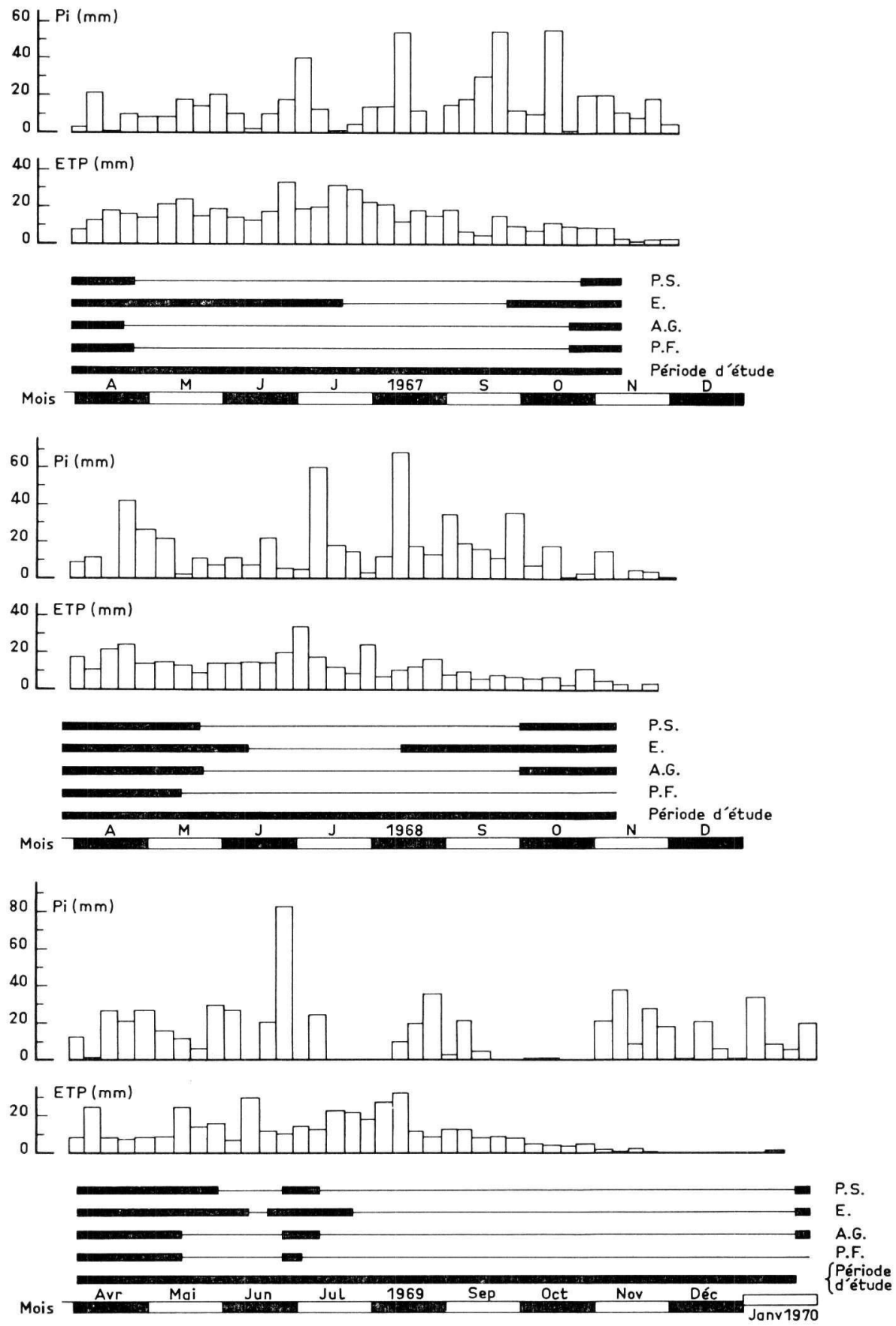

FIG. 7. - Distribution des précipitations, de l'évapotranspiration potentielle, périodes d'absence et de présence des nappes pendant les périodes d'évaluation de l'évapotranspiration potentielle

FIG. 7. - Precipitation, potential evapotranspiration and duration of the water table 
Ils sont percés de nombreux orifices pour permettre la circulation de l'eau. Ces tubes ont été installés dans des troux creusés à l'aide d'une tarière jusqu'à une profondeur de $75 \mathrm{~cm}$, soit $15 \mathrm{~cm}$ en-dessous du niveau moyen de la partie supérieure du pseudogley.

Pour éviter à la surface du sol tout écoulement préférentiel le long des tubes, ces derniers sont entourés d'une collerette de polyéthylène. La profondeur à laquelle se trouve la nappe est relevée deux fois par semaine. Connaissant la profondeur du trou, il est alors possible de calculer l'épaisseur de la couche d'eau. Les fluctuations et l'épaisseur des nappes ne sont pas suffisantes pour résoudre l'équation (2), il faut connaître leur stock d'eau. Compte tenu de la difficulté d'apprécier correctement la porosité (grande variabilité des mesures), il était difficile de se servir directement des valeurs mesurées de ce paramètre pour évaluer le stock d'eau des nappes. Afin de pallier ces difficultés, des profils de sol ont été prélevés dans les peuplements étudiés et reconstitués dans des containers cylindriques de $230 \mathrm{dm}^{3}$ (hauteur $87 \mathrm{~cm}$, diamètre $58 \mathrm{~cm}$ ). Ces contaniers sont pourvus d'un dispositif qui permet de suivre la position des nappes (figure no 2). Après la mise en place des profils, le niveau du sol dans les containers a baissé progressivement par tassement naturel, puis s'est stabilisé au bout d'un an. A ce moment, la remise en place des profils a été estimée convenable et les mesures d'équivalents en hauteur de l'épaisseur des nappes ont pu être effectuées. La porosité baisse ( $\operatorname{tableau}^{\circ} 2$ ) bien sûr avec la profondeur et, pour une fluctuation identique de la nappe, le stock d'eau diminue quand on s'enfonce dans le sol. Il y a une grande variabilité des mesures de porosité pour un même traitement et l'analyse statistique a montré que pour un même niveau les différences entre les traitements n'étaient pas significatives.

Pour évaluer l'importance du drainage, nous avons utilisé un limnigraphe qui a permis d'enregistrer les fluctuations des nappes. Ces fluctuations en période de dessèchement et en l'absence de pluie montrent une phase diurne et une phase nocturne (voir figure $n^{\circ} 3$ ).

En effet, en l'absence de précipitations, l'abaissement de la nappe est conditionné dans la journée par l'évapotranspiration des peuplements et le drainage et la nuit uniquement par le drainage.

L'abaissement des nappes par drainage a pu être mesuré à plusieurs profondeurs. La figure 3 reproduit une partie des enregistrements effectués.

Ainsi, à partir de ces limnigrammes et des mesures d'équivalents en eau en container, il a été possible de calculer les pertes par drainage, niveau par niveau. Dans l'espoir d'améliorer l'analyse de l'évolution des profils hydriques, l'humidité du sol a été suivie à l'aide d'éléments de plâtre (Éléments Gely. Montpellier - France) qui permettent une mesure électrique de l'humidité. Ces éléments ont été installés à une profondeur de 10,30 et $60 \mathrm{~cm}$.

\section{III. - RÉSULTATS}

\section{1. - Fluctuations des nappes perchées dans les peuplements étudiés.}

Les mesures concernant les fluctuations des nappes ont commencé en 1967. Les figures $n^{\circ} 4,5$ et 6 donnent leurs niveaux respectifs pour les périodes comprises entre le moment où le sol est encore à la capacité au champ $\left(^{1}\right.$ ) (au printemps), mais la quitte et le moment où il y revient (en automne ou en hiver). Pour l'année 1967, la période considérée va du 29 mars au 10 novembre, en 1968 du 26 mars au 11 novembre et en 1969 du 2 avril au 21 janvier 1970. Ces dates correspondent au moment où l'ensemble des 4 peuplements se trouvent à la capacité de rétention (figure $\mathrm{n}^{0} \mathrm{7}$ ). Cela était nécessaire pour effectuer les comparaisons entre peuplements. Les périodes de sécheresse peuvent être différentes selon les stations (voir tableau $n^{\circ} 3$ ). L'examen des fluctuations du niveau des nappes montre un cycle saisonnier identique; mais dans le détail, des différences importantes existent : dates d'apparition et de disparition des nappes, profondeur de la nappe au moment de son épaisseur maximum.

En particulier, il y a lieu de remarquer les Épicéas où la nappe disparaît plus tard et apparaît plus tôt que dans les trois autres peuplement étudiés. C'est également chez les Épicéas qu'elle est la plus superficielle au moment de son épaisseur maximum. Les nappes peuvent réapparaître dans le courant de l'été si les pluies sont suffisamment importantes.

La durée de la période sans nappe va en décroissant dans l'ordre suivant : peuplement feuillu, Sapin de Vancouver, Pin Sylvestre, Épicéa. Ce qui suggère bien sûr que les niveaux d'évapotranspiration réelle devraient s'ordonner dans cet ordre.

(1) A la capacité au champ, le pF du sol est compris entre 2,5 et 3,0. 
TABLEAU 3 - TABLE 3

Dates d'apparition et de disparition des nappes dans les peuplements étudiés

The date of apparition and disappearance of water table

\begin{tabular}{|c|c|c|c|c|c|c|c|c|c|}
\hline \multirow{2}{*}{ Essence } & \multicolumn{3}{|c|}{1967} & \multicolumn{3}{|c|}{1968} & \multicolumn{3}{|c|}{1969} \\
\hline & Disparition & Apparition & $\begin{array}{l}\text { Nbre jours } \\
\text { sans nappes }\end{array}$ & Disparition & Apparition & $\begin{array}{l}\text { Nbre jours } \\
\text { sans nappes }\end{array}$ & Disparition & Apparition & $\begin{array}{l}\text { Nbre jours } \\
\text { sans nappes }\end{array}$ \\
\hline Épicéa $\ldots \ldots \ldots \ldots$ & 18 juillet & 26 septembre & 70 & 11 juin & 12 août & 62 & $\begin{array}{l}11 \text { juin } \\
23 \text { juillet }\end{array}$ & $\begin{array}{l}18 \text { juin } \\
21 \text { janvier } \\
1970\end{array}$ & $\begin{array}{r}7 \\
182\end{array}$ \\
\hline Pin sylvestre $\ldots \ldots \ldots$ & 25 avril & 24 octobre & 182 & $21 \mathrm{mai}$ & $1^{\mathrm{er}}$ octobre & 133 & $\begin{array}{l}21 \text { mai } \\
9 \text { juillet }\end{array}$ & $\begin{array}{l}25 \text { juin } \\
21 \text { janvier } \\
1970\end{array}$ & $\begin{array}{r}4 \\
196\end{array}$ \\
\hline Sapin de Vancouver & 19 avril & 20 octobre & 184 & $21 \mathrm{mai}$ & $1^{\mathrm{er}}$ octobre & 173 & $\begin{array}{l}14 \text { mai } \\
9 \text { juillet }\end{array}$ & $\begin{array}{l}25 \text { juin } \\
21 \text { janvier } \\
1970\end{array}$ & $\begin{array}{r}42 \\
196\end{array}$ \\
\hline Feuillu $\ldots \ldots \ldots \ldots$ & 25 avril & 20 octobre & 178 & $14 \mathrm{mai}$ & $\begin{array}{l}8 \text { janvier } \\
1969\end{array}$ & 239 & $\begin{array}{l}14 \text { mai } \\
2 \text { juillet }\end{array}$ & $\begin{array}{l}25 \text { juin } \\
21 \text { janvier } \\
1970\end{array}$ & $\begin{array}{r}42 \\
203\end{array}$ \\
\hline
\end{tabular}




\section{2. - Variations de l'humidité des sols des peuplements étudiés.}

L'état d'humidité des sols a été suivi à l'aide d'éléments de plâtre (Éléments Gely. Montpellier - France). En fait, compte tenu de la précision de ces appareils, il s'agit plutôt de mesures qualitatives que quantitatives. Ces éléments ont permis de déterminer en parallèle avec les fluctuations des nappes, les périodes où les sols étaient à la capacité de rétention et les périodes d'assèchement important. A titre d'exemple, voir les graphiques $n^{0} 8$. En fait,
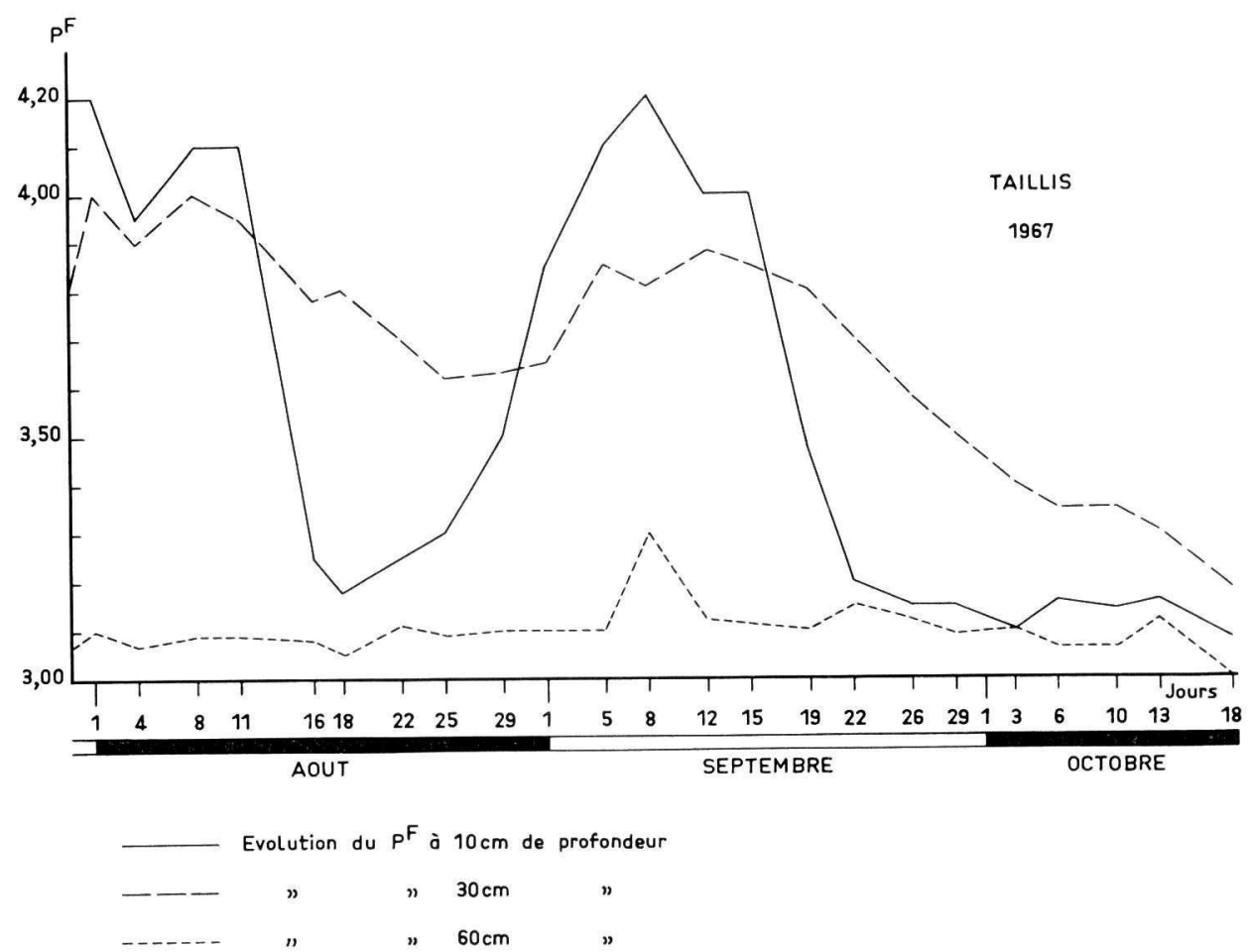

FIG. 8. - Évolution du potentiel capillaire en août-septembre-octobre 1967 $8 \mathrm{~A}$ : Taillis (Feuillus)

FIG. 8. - Variation of the soil water potential in August, September and October 1967

l'utilisation des sondes n'a été possible que pendant l'année qui a suivi leur installation. L'année suivante, de nombreuses irrégularités sont apparues et finalement il n'a pas été possible d'utiliser valablement ces appareils.

A la lumière de cette expérience, il apparaît que ces sondes permettent seulement de déterminer si un sol est humide ou non. Il est illusoire de vouloir apprécier quantitativement des différences d'humidité.

\section{3. - Évaluation de l'évapotranspiration réelle (ETR) des peuplements étudiés.}

Les valeurs mensuelles des précipitations et de l'évapotranspiration potentielle au poste climatologique sont données par le tableau no 4 .

Les calculs de ETR ont été effectués en utilisant l'équation 2. Le tableau $\mathrm{n}^{0} 5$ donne 


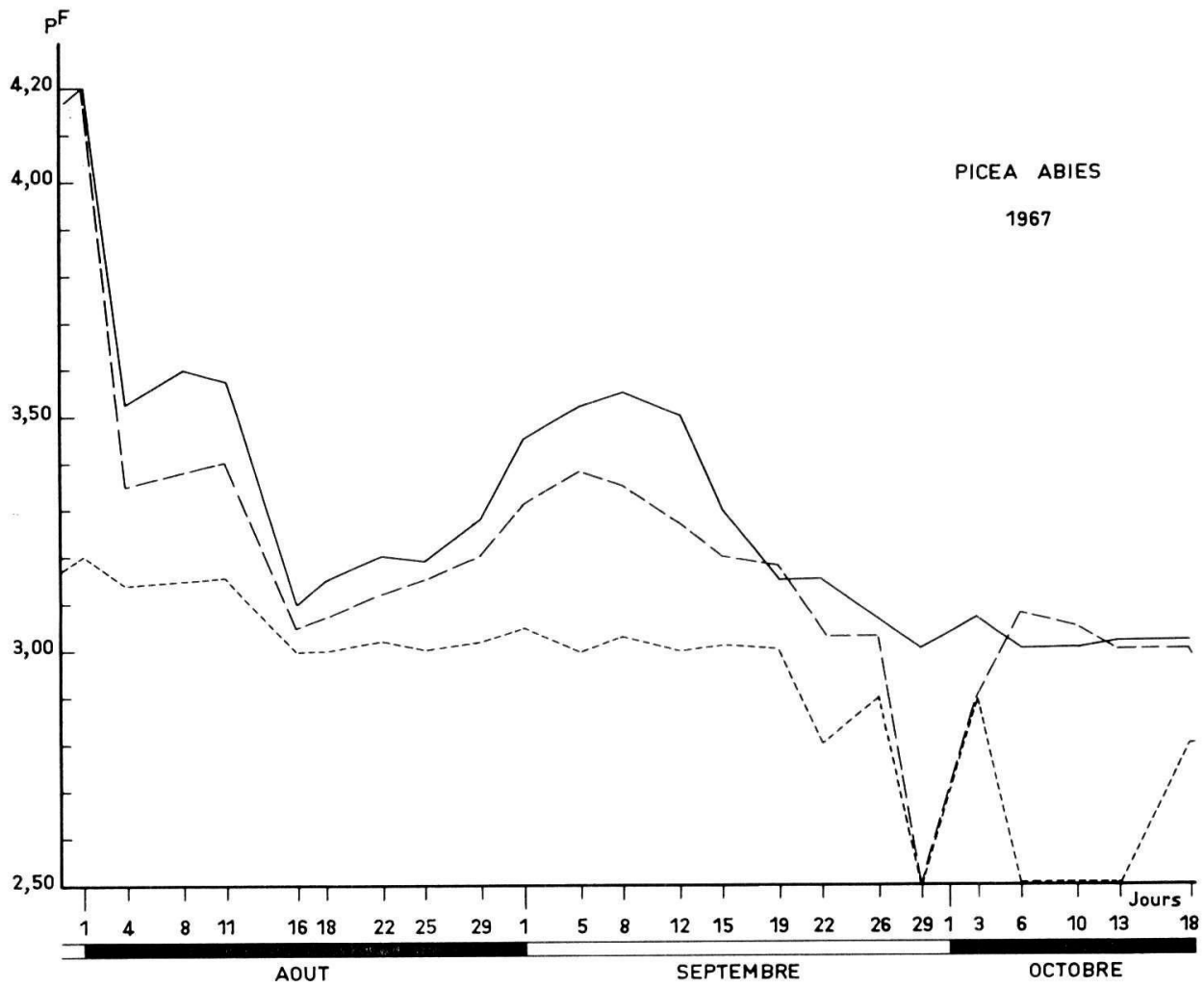

Evolution du $\mathrm{PF}^{\mathrm{F}}$ ò $10 \mathrm{~cm}$ de profondeur

\begin{tabular}{|c|c|c|c|}
\hline--- & $"$ & $"$ & $30 \mathrm{~cm}$ \\
\hline$\cdots \cdots$ & $"$ & $n$ & $60 \mathrm{~cm}$ \\
\hline
\end{tabular}

FIG. 8. - Évolution du potentiel capillaire en août-septembre-octobre 1967 $8 B$ : Picea abies

FIG. 8. - Variation of the soil water potential in August, September and October 1967

pour les périodes considérées les valeurs de l'évapotranspiration potentielle $\left({ }^{1}\right)$ (ETP) et du rapport $\frac{\text { ETR }}{\text { ETP }}$.

(1) L'évapotranspiration potentielle a été évaluée à l'aide de la formule de BoucheT (1964) ETP = $\alpha[1+\lambda(\theta)] \mathrm{E} p$ avec

$-\alpha=0,37$

- $\mathrm{E} p=$ évaporation mesurée en mm sous-abri à l'évaporomètre Piche

$-[1+\lambda(\theta)]$ coefficient de correction de l'évaporation sous-abri

$$
\begin{aligned}
\theta \text { température }= & \theta \mathrm{m}+\frac{\theta \mathrm{M}-\theta \mathrm{m}}{4} \\
& \theta \mathrm{m}: \text { température minimum } \\
& \theta \mathrm{M}: \text { température maximum } \mid \text { sous-abri }
\end{aligned}
$$

Les calculs ont été effectués à partir des données recueillies à un poste climatologique situé à $500 \mathrm{~m}$ des peuplements.

Remarquons que cette formule ne permet pas d'apprécier très précisément l'ETP, mais qu'elle doit être considérée comme un index du pouvoir évaporant de l'air. 


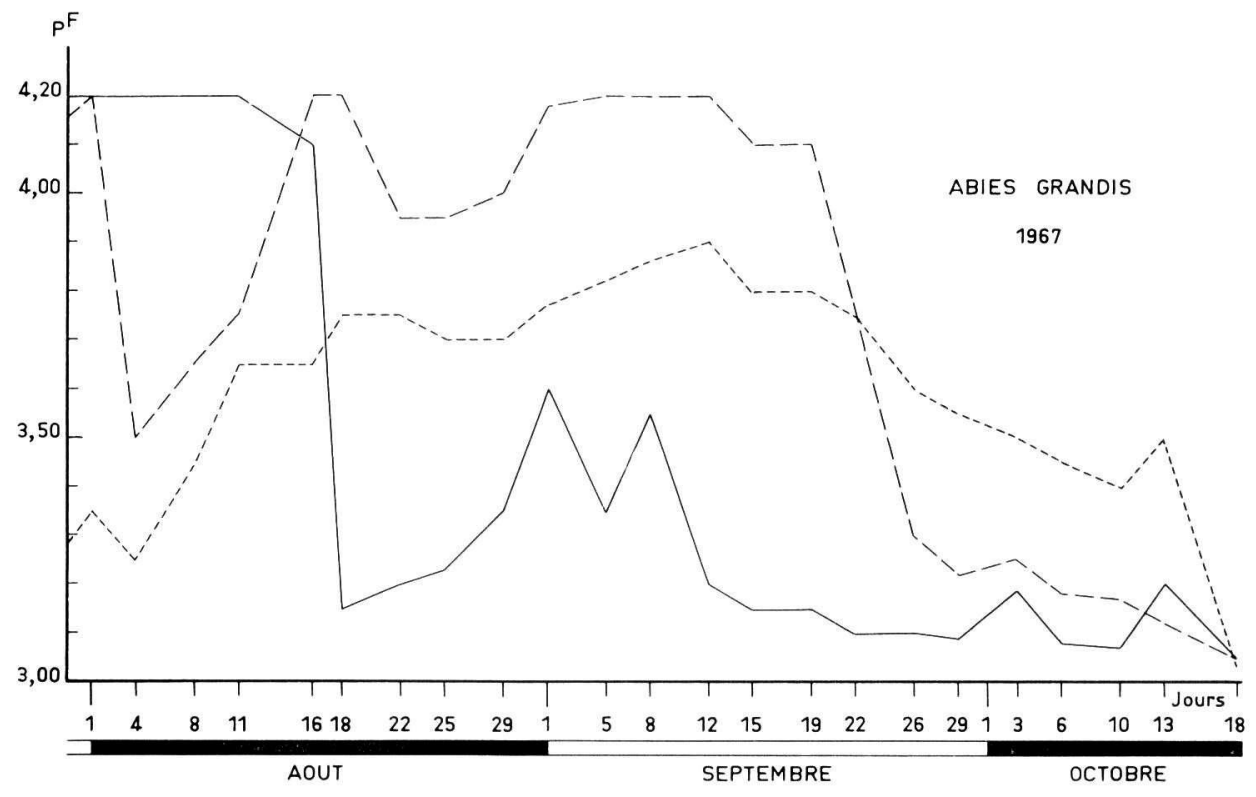

Evolution du $P^{F}$ à $10 \mathrm{~cm}$ de profondeur

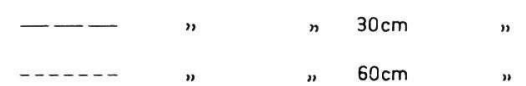

FIG. 8. - Evolution du potentiel capillaire en â̂ut-septembre-octobre 1967. $8 \mathrm{C}$ : Abies grandis

FIG. 8. - Variation of the soil water potential in August, September and October 1967

Le peuplement d'Épicéa est celui qui évapotranspire le moins sur les 3 années de mesure. Bien qu'en 1967 les Pins sylvestres aient évapotranspiré le plus, ils n'arrivent qu'en $3^{e}$ position les années suivantes (1968 et 1969), derrière le peuplement feuillu et les Sapins de Vancouver. Le rapport ETR/ETP est dans l'ensemble assez voisin de 1,0 puisqu'il varie au total entre 0,93 et 1,30 .

\section{IV. - DISCUSSION ET CONCLUSION}

Les résultats obtenus montrent qu'il existe des différences relativement importantes dans les évaluations de l'évapotranspiration réelle des peuplements étudiés. En particulier, les Épicéas évapotranspirent notablement moins que les autres. C'est d'ailleurs ce que suggérait l'examen des figures rapportant les fluctuations des nappes perchées. Il y a lieu de se demander comment ces différences peuvent être expliquées. En premier lieu, il faut remarquer (tableau $n^{0}$ 1) que les Épicéas sont les plus jeunes des peuplements étudiés. Or, il semble maintenant bien établi (Molchanov 1963) que l'évapotranspiration réelle des peuplements augmente avec l'âge jusqu'à un maximum qui varierait selon les essences et la station, mais qui en première approximation se situerait entre 60 et 80 ans. En ce qui concerne le peuplement feuillu, il faut dire aussi qu'il est issu d'un taillis, donc que son système racinaire est 
TABLEAU 4 - TABLE 4

Précipitations ( $\mathrm{mm}$ ) et évapotranspiration potentielle $(\mathrm{mm})$ au poste climatologique d'Amance pour les années 1967-68-69

Precipitation and potential evapotranspiration from the climatologic station Amance

\begin{tabular}{|c|c|c|c|c|c|c|}
\hline \multirow{2}{*}{$\begin{array}{l}\text { Année } \\
\text { Mois }\end{array}$} & \multicolumn{2}{|l|}{1967} & \multicolumn{2}{|l|}{1968} & \multicolumn{2}{|l|}{1969} \\
\hline & Précipitations & ETP & Précipitations & ETP & Précipitations & ETP \\
\hline 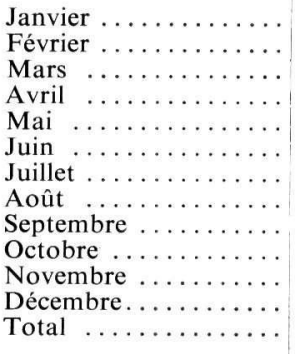 & $\begin{array}{r}37,4 \\
43,9 \\
49,2 \\
36,5 \\
71,9 \\
41,8 \\
57,8 \\
91,5 \\
123,4 \\
89,3 \\
61,3 \\
51,5 \\
755,5\end{array}$ & $\begin{array}{c}10,3 \\
25,5 \\
51,1 \\
62,5^{*} \\
81,1^{*} \\
80,2^{*} \\
112,8^{*} \\
71,2 \\
44,1 \\
40,5 \\
16,3 \\
7,5 \\
603,1\end{array}$ & $\begin{array}{r}64,0 \\
59,8 \\
41,5 \\
73,0 \\
56,6 \\
46,2 \\
99,3 \\
129,2 \\
96,1 \\
33,5 \\
24,9 \\
54,1 \\
778,2\end{array}$ & $\begin{array}{l}6,8 \\
12,3 \\
46,5^{*} \\
78,0^{*} \\
56,1 \\
77,1^{*} \\
78,5 \\
51,6 \\
36,7 \\
28,4 \\
10,9 \\
4,2 \\
487,1\end{array}$ & $\begin{array}{r}30,9 \\
45,0 \\
78,9 \\
76,0 \\
73,1 \\
125,4 \\
25,0 \\
67,1 \\
30,9 \\
3,4 \\
95,7 \\
47,5 \\
698,9\end{array}$ & $\begin{array}{r}11,6 \\
6,3 \\
22,3 \\
51,9 \\
71,3 \\
64,7 \\
83,5^{*} \\
85,7^{*} \\
45,3^{*} \\
22,0^{*} \\
8,7 \\
0,8 \\
474,1\end{array}$ \\
\hline
\end{tabular}

* mois où ETP est supérieure aux précipitations.

plus vieux que lui et de ce fait, les possibilités « d'extraction » de l'eau du sol sont peut-être supérieures à celles d'un peuplement de franc pied du même type.

Certains nous diront que malgré la présence du pseudogley, des racines arrivent à s'enfoncer dans le sous-sol marneux et s'alimentent ainsi en eau. C'est peu probable pour les peuplements étudiés et particulièrement pour l'Épicéa, qui a un enracinement superficiel.

Quoi qu'il en soit, nous aurions alors une évaluation par défaut (l'écart avec la valeur réelle ne peut pas être considérable, compte tenu du fait que nous dépassons l'évaluation de l'évapotranspiration potentielle par suite de la consommation d'énergie latérale).

En effet, on remarquera aussi que l'hétérogénéité de la couverture végétale intervient dans les phénomènes d'évapotranspiration et les apports latéraux d'énergie peuvent faire varier considérablement l'évapotranspiration potentielle d'un point à un autre. Les apports d'énergie par advection peuvent être non négligeables dans le cas où les peuplements se trouvent à proximité d'une zone agricole (céréales par exemple) ou d'un terrain déboisé, qui présentent en été une évapotranspiration potentielle plus élevée que la forêt voisine. Les apports d'énergie se font évidemment de la zone qui évapore le moins vers celle qui évapore le plus. La captation de cette énergie advective sera d'autant plus importante que les peuplements auront une taille plus élevée. Le peuplement feuillu se trouve sous le vent d'une grande zone récemment déboisée fonctionnant en "source chaude ». La consommation d'eau de ce peuplement peut donc dépasser, grâce à l'énergie d'avection, l'équivalent en eau du rayonnement net. Le peuplement d'Abies grandis a une taille nettement plus élevée que les autres peuplements et il est également possible que cela intervienne dans l'explication des différences constatées. 
TABleAU 5 - TABLE 5

Évapotranspiration réelle des peuplements étudiés (ETR), évapotranspiration potentielle (ETP), précipitations incidentes ( $P i$ ) et rapport $\frac{E T R}{E T P}$ pour les périodes étudiées (valeurs en $\mathrm{mm}$ )

Real Evapotranspiration (ETR) from stands, potential evapotranspiration (ETP) precipitation and $\frac{E T R}{E T P}$ for diferent periods

\begin{tabular}{|c|c|c|c|c|c|c|c|c|c|c|}
\hline \multirow{2}{*}{ Période } & \multirow{2}{*}{$\mathrm{Pi}$} & \multirow{2}{*}{ ETP } & \multicolumn{4}{|c|}{ ETR } & \multirow{2}{*}{$\begin{array}{c}\frac{E T R}{\text { ETP }} \\
(1)\end{array}$} & \multirow{2}{*}{$\begin{array}{c}\text { ETR } \\
\text { ETP } \\
(2)\end{array}$} & \multirow{2}{*}{$\begin{array}{c}\text { ETR } \\
\text { ETP } \\
(3)\end{array}$} & \multirow{2}{*}{$\begin{array}{r}\frac{\text { ETR }}{\text { ETP }} \\
(4)\end{array}$} \\
\hline & & & $\begin{array}{l}\text { Épicéa } \\
\text { (1) }\end{array}$ & $\begin{array}{l}\text { Pin } \\
\text { Sylvestre } \\
\text { (2) }\end{array}$ & $\begin{array}{l}\text { Sapin de } \\
\text { Vancouver } \\
\text { (3) }\end{array}$ & $\begin{array}{c}\text { Feuillu } \\
(4)\end{array}$ & & & & \\
\hline 29 mars 1967 au 10 novembre $1967 \ldots$ & 532,6 & 502,1 & 465,8 & 516,5 & 497,5 & 515,9 & 0,93 & 1,03 & 0,99 & 1,02 \\
\hline 26 mars 1968 au 11 novembre $1968 \ldots$ & 548,7 & 410,0 & 428,7 & 472,8 & 524,9 & 536,8 & 1,04 & 1,15 & 1,28 & 1,30 \\
\hline 2 avril 1969 au 21 janvier $1970 \ldots \ldots$ & 586,8 & 437,0 & 509,0 & 525,9 & 556,0 & 560,2 & 1,16 & 1,20 & 1,27 & 1,28 \\
\hline
\end{tabular}


L'interception représente un paramètre important susceptible d'expliquer une partie des différences observées. L'évapotranspiration totale d'un peuplement peut s'écrire :

$\mathrm{ETR}$ totale $=$ ETR peuplement $+\mathbf{I} n$.

In : eau interceptée par le couvert peut diminuer plus ou moins ETR peuplement et par voie de conséquence influencer ETR totale. Dans des recherches effectuées sur des arbres isolés (AussenAC, résultats non encore publiés) en simulant les phénomènes d'interception par des pulvérisations d'eau, nous avons trouvé que $90 \%$ de l'eau interceptée était évaporée sans contrepartie pour les plants. Les plants traités ont transpirés $30 \%$ de moins que les plants témoins. Mais, la consommation totale d'eau (transpiration + interception) a été finalement 3,2 fois plus grande. RutTer (1964), THORUD (1967) ont trouvé des résultats voisins. Il est probable cependant que dans le cas de peuplements $\left({ }^{1}\right)$ étendus et à couvert fermé, ces pourcentages de réduction de la transpiration sont différents. Cette réduction de transpiration par l'eau interceptée est variable et dépend, toutes choses égales par ailleurs, non seulement de la morphologie des feuilles, mais aussi de la structure de la forêt. Ainsi, si l'on considère sous cet angle les parcelles étudiées, on remarque que le peuplement d'Épicéa est le plus dense et que dans ce cas précis, compte tenu de la réduction de la vitesse du vent (plus importante que dans les autres cas), la réduction de sa transpiration doit être particulièrement forte. Il est alors clair qu'une étude globale des phénomènes n'est pas suffisante pour leur bonne compréhension (Aussenac 1970).

Quoi qu'il en soit, nos études permettent malgré tout d'avoir des données raisonnablement approchées sur les niveaux évapotranspiratoires des peuplements forestiers.

On notera que les chiffres obtenus à Nancy sont voisins de ceux rapportés par divers auteurs. Rutter (1964) en Angleterre pour un peuplement de Pinus silvestris L. trouve que le rapport $* \frac{\text { ETR }}{\text { ETP }}$ est compris entre 0,98 et 1,20. FouRT et HINSON (1970) donnent 1,00 à 1,47 pour le Pinus nigra ssp. Laricio et 0,64 à 0,94 pour Pseudotsuga menziessii.

L'évaluation de l'évapotranspiration des peuplements forestiers est un problème difficile. Plusieurs techniques sont utilisées, ce qui montre bien sûr que pour l'instant aucune

(1) Il est possible d'aborder le problème de façon théorique comme l'a fait RUTTER (1968) en partant des équations de Burgy et Pomeroy (1958). (Perte nette par interception $=\left(1-\mathrm{E}_{\mathrm{T}} / \mathrm{E}_{1}\right) \mathrm{I}$ avec $\mathrm{I}$ hauteur d'eau interceptée, $E_{T}$ taux d'évapotranspiration, $E_{1}$ taux d'évaporation de l'eau interceptée) et de MONTEITH (1965):

et d'écrire alors que :

$$
\lambda \mathrm{E}=\frac{s \mathrm{R}_{n}+\mathrm{c}\left[e_{s}(\mathrm{~T})-e\right] / r_{a}}{s+\gamma\left[\left(r_{a}+r_{s}\right) / r_{a}\right]}
$$

Perte nette par interception $=\frac{\gamma r_{s} \mathbf{I}}{(\mathrm{S}+\gamma) r_{a}+\gamma r_{s}}$

avec :

$\gamma$ constante psychrométrique

I hauteur d'eau interceptée

$S$ pente de la courbe de pression de vapeur saturante

$r_{s}$ résistance interne des arbres à la diffusion de l'eau en $\mathrm{sec} / \mathrm{cm}^{-1}$

$r_{s}$ résistance à la diffusion de l'eau dans l'air au-dessus des surfaces foliaires en $\mathrm{sec} / \mathrm{cm}^{-1}$.

A partir de cette formule, il est visible que la perte par interception sera d'autant plus élevée et donc voisine de 1 que $r_{8}$ sera grand devant $r_{a}$. Or, $r_{s}$ varie avec les essences et $r_{a}$ diminuerait avec la hauteur de végétation. Il est donc clair que la perte nette de l'interception doit varier avec les essences et les types de peuplements et bien sûr ceci a une incidence importante sur le bilan évapotranspiratoire.

* ETP est ici calculé à partir de l'évaporation d'une nappe libre en bac. 
n'est vraiment satisfaisante (AUSSENAC 1970). La méthode que nous avons utilisée ici fait partie des méthodes dites globales, qui essayent de chiffrer en bloc l'évapotranspiration totale du peuplement considéré.

L'intérêt de notre technique, originale à notre connaissance, est d'avoir utilisé les nappes perchées. En effet, les éléments de plâtre (éléments GELY) n'étaient pas suffisants pour permettre de déterminer avec précision le moment où le sol était à la capacité au champ. L'intérêt de ces appareils nous apparaît d'ailleurs très limité pour ce type de recherche. De toute façon, l'utilisation du bilan d'eau du sol ne peut être valablement envisagée que quand la profondeur utilisable du sol est bien connue.

D'une façon générale, il apparaît que sous les climats du type de celui de Nancy (tempéré humide) et pour des sols identiques aux nôtres, les peuplements forestiers à couvert fermé et à structure comparable ont un niveau évapotranspiratoire voisin; cette évapotranspiration réelle est égale ou supérieure à l'évapotranspiration potentielle. Apparemment, ils sont donc dans de bonnes conditions pour utiliser pleinement leurs possibilités intrinsèques de production de bois (du moins lorsqu'on raisonne globablement sur la période de végétation entière)... On sait qu'il n'en est pas toujours ainsi dans d'autres régions en France et même en Lorraine sur des sols moins favorables.

Mais, si l'on raisonne au niveau élémentaire de la journée, le problème est beaucoup plus complexe, car comme nous l'avons dit précédemment, la part réelle de la perte par interception peut être très variable et il peut y avoir alors une "consommation de luxe " très importante qui masque l'évapotranspiration réelle du peuplement, ce dernier pouvant alors être dans des conditions difficiles pour ce qui est du rapport ETR/ETP. En effet, les recherches faites ces dernières années (BOUCHET, 1964) montrent qu'il faut considérer le rapport Évapotranspiration potentielle/eau susceptible d'être fourni par le système végétal sol, de façon quasi instantanée, comme particulièrement important pour la compréhension des phénomènes d'évapotranspiration. Dans la mesure où l'offre est inférieure à la demande, il y a régulation stomatique plus ou moins importante et donc par voie de conséquences baisse de la photosynthèse. Ainsi, il est certain que ces phénomènes ne pourront être clairement compris que dans la mesure où l'on aura précisé d'une part le rôle exact de l'interception et d'autre part, les relations ETR/ETP à l'échelle de la journée.

TABLEAU 6 - TABLE 6

Efficience de l'eau dans les peuplements étudiés

Efficiency of water from the four stands

\begin{tabular}{|c|c|c|c|c|}
\hline Essences & Épicéa & Pin sylvestre & Abies grandis & $\begin{array}{l}\text { Peuplement } \\
\text { feuillu }\end{array}$ \\
\hline Consommation moyenne d'eau . & 467,80 & 505,0 & 526,1 & 537,6 \\
\hline $\begin{array}{l}\text { Rapport } \\
\text { ETR/Accroissement courant } \mathrm{m}^{3} / \text { ha/an }\end{array}$ & 18,4 & 45,0 & 17,5 & 68,9 \\
\hline
\end{tabular}


Il est possible de calculer pour les 3 années considérées la consommation moyenne d'eau des peuplements étudiés. Le tableau n 1 donne 1 'accroissement courant en $\mathrm{m}^{3} / \mathrm{ha} / \mathrm{an}$ de bois fort. Il existe une relation étroite entre l'accroissement de biomasse et l'accroissement en volume bois fort et il est intéressant d'utiliser ce dernier pour avoir une valeur approchée de l'efficience de l'eau dans les 4 peuplements (Tableau 6). Nous remarquons que le peuplement feuillu et les pins sylvestres ont une mauvaise efficience de l'eau par comparaison avec les Épicéas et les Abies grandis. Ces résultats nous paraissent intéressants, car ils montrent que pour des consommations d'eau voisines, il est possible d'avoir des productions $\left(\mathrm{m}^{3}\right)$ de bois différentes. Ainsi, l'épicéa se présente comme une essence très efficace, il en est de même pour l'Abies grandis qui pouvait poser un problème, car il s'agit d'une essence exotique et l'on se demandait si son introduction ne risquait pas de perturber le cycle hydrologique. Ces conclusions, bien sûr, ne sont valables que pour des types de station identiques aux nôtres.

Reçu pour publication en mai 1972.

\section{REMERCIEMENTS}

Nous tenons à remercier particulièrement :

- Monsieur Dematieu, Maître Assistant à la Faculté des Sciences de Nancy, qui nous a prêté une partie du matériel nécessaire à l'expérimentation.

- Monsieur Bouchet R.-J., Directeur de Recherches à la Station Centrale de Bioclimatologie Agricole, et Monsieur PARdÉ J., Directeur de la Station de Sylviculture et de Production, dont les conseils et les critiques ont contribué à la mise en forme définitive de cet article.

\section{SUMMARY}

\section{THE ACTUAL EVAPOTRANSPIRATION OF FOUR FOREST STANDS}

IN THE EAST OF FRANCE

The actual evapotranspiration (ETR) of four forest stands, a Scotch Pine (Pinus silvestris), a Spruce (Picea Abies), a Fir (Abies grandis) and a harwood stand (Fagus silvatica + Carpinus betulus) has been studied during three years from 1967 on to 1969.

These high forest had a regular structure and are located upon a marbled and lixiviated brown soil with a seasonal watersheet (at $60 \mathrm{~cm}$ depth). Evapotranspiration has been estimated between periods at which soil moisture reaches the field capacity (start and end of the vegetation period). The basic equation of the method is :

$$
\begin{aligned}
\mathrm{ETR} & =\mathrm{P}_{i}-\mathrm{D} \pm \Delta \mathrm{N} \\
\mathrm{P}_{i} & =\text { Incident rainfall on the stand } \\
\mathrm{D} & =\text { Lateral and vertical drainage } \\
\Delta \mathrm{N} & =\text { Level fluctuations of the perched watersheet. }
\end{aligned}
$$

Diurnal and nocturnal watertable fluctuations measured in piezometric pipes allowed drainage estimations. The results show differences between stands. The Spruce stand for instance evapotranspirated less than the other stands. Different age, different structure and different environmental conditions (advection) can explain this situation.

Rainfall interception must also be considered, as the net loss of water can be quite different according to the species and the stand structure.

This study shows that the actual evapotranspiration is higher or equal to the potential evapotranspiration calculated with the Boucher equation. 
The relatively small differences recorded seem to indicate that under temperate climates and on soils identical to ours, closed stands of similar age and structure have almost the same actual evapotranspiration.

\section{ZUSAMMENFASSUNG}

\section{UNTERSUCHUNG DER REELLEN EVAPOTRANSPIRATION VON VIER BEST “ NDEN IN OSTFRANKREICH}

In vier einschichtigen Hochwaldbeständen, einem Kiefernbestand (Pinus sylvestris), einem Fichtenbestand (Picea abies), einem Küstentannenbestand (Abies grandis) und einem Buchen -Hainbuchen Mischbestand (Fagus silvatica und Carpinus betulus) wurde der Wasserverbrauch während dreier Vegetationsperioden (1967-1969) untersucht.

Die Bestände haben eine einfache Struktur und stocken in ebenem Gelände auf marmorisierter und lessivierter Braunerde mit einem Stauhorizont in ca $60 \mathrm{~cm}$ Tiefe.

Es wird versucht, die reelle Evapotranspiration (E.T.R.) der Bestände für jene Perioden zu ermitteln, während welcher der Boden zu Beginn (Anfang der Vegetationsperiode) und am Ende (Ende der Vegetationsperiode) einen der Feldkapazität entsprechenden Wassergehalt aufweist. Es wurde folgende einfache Grundgleichung verwendet :

$$
\begin{aligned}
& \text { E T R }=P_{i}-\mathrm{D} \pm \Delta N \\
& P_{i}=\text { einfallender Niederschlag (Freiland) } \\
& \mathrm{D}=\text { laterale und vertikale Drainage } \\
& \Delta \mathrm{N}=\text { Schwankungen der Stauwassermenge. }
\end{aligned}
$$

Die Drainage (D) wurde durch die Beobachtung der Tages und Nachtschwankungen des Stauwasserspiegels ermittelt. Die Schwankungen der Stauwassermenge $(\Delta N)$ wurden mit Hilfe von Rohrpegelmessungen (tube piezométrique) verfolgt. Die Ergebnisse lassen Unterschiede zwischen den Beständen erkennen. In sbesondere hat der Fichtenbestand eine geringere Evapotranspiration als die anderen Bestände. Dies lässt sich durch Unterschiede des Alters und der Struktur und der Ökologischen Situation (Advektion) erklären.

Es kann als sicher angenommen werden, dass die Interzeption gleichfalls eine bedeutende Rolle spielt, denn der Nettointerzeptionsverlust weist je nach Holzart und Bestandesstruktur sehr grosse Unterschiede auf.

Die vorliegende Untersuchung zeigt, dass die reelle Evapotranspiration (E T R) grösser oder gleichhoch ist, als die, nach der Formel von BouchET ermittelte, potentielle Evapotranspiration (E T P).

Die verhältnismässig geringen Unterschiede der E T R zwischen den Beständen scheinen anzudeuten, dass in unseren feuchttemperierten Klimaten auf vergleichbaren Standorten und Beständen mit Kronenschluss, die reelle Evapotranspiration der verschiedenen Holzarten keine grossen Unterschiede aufweist.

\section{RÉFÉRENCES BIBLIOGRAPHIQUES}

AussenaC G., 1968. - Interception des précipitations par le couvert forestier. Ann. Sci. forest, 25, (3), 135-156.

Aussenac G., 1970. - Action du couvert sur la distribution au sol des précipitations Ann. Sci. forest, 27, (4), 389-399.

AussenaC G., 1970. - Aperçu du rôle de la forêt dans l'économie de l'eau. R.F.F. XXII, 6, 603-618.

Aussenac G. - Étude des effets de l'eau interceptée sur la transpiration de l'Épicéa (Picea abies) cultivé en pot (en préparation).

Bouchet R.-J., 1964. - Evapotranspiration réelle, évapotranspiration potentielle et production agricole. Ann. agron., 4, 5, 743-824.

Fourt D.F. - Hinson W. H., 1970. - Water Relations of tree crops. A comparison between Corsican Pine and Douglas fir in Southeast England. J. appl. Ecol., 7, 2, 295-309.

LeVy G., 1969. - Premiers résultats d'étude comparée de la nappe temporaire des pseudogleys sous résineux et sous feuilles. Ann. Sci. forest., 65-79. 
Molchanov A.-A., 1963. - The hydrological role of forest. Jerusalem. Israël Programm for Scientific Translation, $405 \mathrm{p}$.

Rutter A. J., 1964. - Studies in the water relations of Pinus sylvestris in Plantation conditions. II The annual cycle of soil moisture change and derived estimates of evaporation. J. appl. Ecol., 29-44.

RutTer A.-J., 1968. - Water consumption by forests. Water deficit and plant growth New-York. Academic Press, 23-84.

THORUD, 1967. - The effect of applied interception on transpiration rates of potted Ponderosa Pine. Water Resources Res. 3, 2, 443-450. 